\title{
Color Image Segmentation using Wavelet
}

\author{
Samer Kais Jameel \\ Aurangabad, Dr.B.A.M.U, \\ Department of computer \\ Science and IT
}

\author{
Ramesh R.Manza \\ Aurangabad, Dr.B.A.M.U, \\ Department of computer \\ Science and IT
}

\begin{abstract}
In this paper, we discussed color image segmentation by extract the optimal features with which to discriminate between regions. Many real or texture images are made up of smooth regions and are best segmented using features in different areas. Schemas that select the optimal features for each pixel using wavelet analysis are proposed, leading to robust segmentation algorithm. Using two dimensions wavelet transforms to decompose the image into subbands channels and made up the of smooth image and convert the image into NTSC color space enables us to quantify the visual differences in the image, and then applies a clustering technique to partition the image into a set of "homogeneous" regions is also proposed.
\end{abstract}

\section{Keywords}

Segmentation, color image, wavelet transform, k-means clustering.

\section{INTRODUCTION}

Image segmentation is becoming increasingly important in a variety of fields such as video coding, computer vision, and medical imaging [1], [2]. The goal of segmentation is to simplify and/or change the representation of an image into something that is more meaningful and easier to analyze [3]. The objective of dividing an image into homogeneous region remains a challenge, especially when the image is made up of complex textures. Image segmentation is becomes much simpler for images made up of smoother regions, However, many real images are made up of a variety of smooth regions, which need to be reliably identified in the segmentation algorithm. Successfully segmenting only the smooth regions depending on feature used [4], therefore, it would clearly be desirable to have some means of feature selection prior to segmentation. In this way smooth region can be segmented using means and variance. A schema that made up of smooth images and selects optimal features using wavelet analysis is proposed. The algorithm is:

\section{- Step 1: Read Image}

- Step 2: Analysis the image using wavelet transforms at level 2.

- Step 3: Convert Image from RGB Color Space to NTSC Color Space

- Step 4:Classify the Colors in I and Q Space Using K-means clustering.

- Step 5: Label Every Pixel in Image Using the Results from K-means.

The images represent color as RGB values directly in an RGB image, or indirectly in an index image, where the color map is stored in RGB format. However, there are other color spaces also called color models whose use in some application may be more convenient and/or appropriate [3]. In this paper we convert the image from RGB space into NTSC also known as YIQ color space enables us to quantify the visual differences in the image, image data consist of three components luminance $(\mathrm{Y})$, hue $(\mathrm{I})$, and saturation $(\mathrm{Q})$.

Clustering techniques [5] are commonly used for image segmentation in a multidimensional feature space. The widely used of K-means clustering usually require a threshold in its determination of the optimal number of regions for segmentation.

The block diagram in Fig. 1 summarizes the main features of the proposed segmentation algorithm.

\begin{tabular}{|c|c|c|c|}
\hline $\begin{array}{l}\text { ORIGINAL } \\
\text { IMAGE }\end{array}$ & $\begin{array}{l}\text { WAVELET } \\
\text { ANALYSIS }\end{array}$ & CLUSTERI & SEGMENTA \\
\hline
\end{tabular}

Fig.1 Block diagram of the propose segmentation algorithm.

\section{PRINCIPAL OF SEGMENTATION}

The stage of image segmentation usually involves the development of the feature space. This comprises clustering the value of features of each pixel (or block of pixel) in the image each feature should in same way describe the appearance of the local area surrounding the pixel. These features make up the feature vector for each pixel so that each is represented by a point in multidimensional feature space. Consequently, if the features used for the image are good descriptors similar appearing region in the image will contain pixels whose feature vectors occupy similar position in the feature space. There is known as clusters, and the purpose of clustering is to identify these clusters and classify the image's pixels accordingly. The quality and the accuracy of segmentation ultimately depend on the type of feature used therefore, it is very important that these features suitably characterize the aspects of the image on which the segmentation is to be used. For example images made up of smooth regions can more easily be segmented using mean and variance as feature. As a result, existing segmentation algorithms have failed to produce meaningful segmentation of many real images. The proposed algorithm involve the used of wavelet analysis to determine the optimal feature corresponding to each pixel or block of pixels.

\section{WAVELET ANALYSIS OF IMAGE}

A wavelet is a wave-like oscillation with an amplitude that starts out at zero, increases, and then decreases back to zero. It can typically be visualized as a "brief oscillation" like one might see recorded by a seismograph or heart monitor [3].Wavelet analysis provides a more formal, precise, and 
unified approach to multiresolution representations [6], the 2D wavelet transform [7], [8] is a very popular tool in image processing. To achieve a high analysis of the image, it is necessary to choose the best wavelet filter bank and decomposition level, which will pay a crucial role in analysis the image. Wavelet filters plays a crucial part in achieving an effective coding performance, because there is no filter that performs the best for all images [9]. Because orthogonal filters have a property of energy preservation whereas, biorthogonal filters lack it [10]. The choice of optimal wavelets has several criteria. The main criteria are:

$$
\begin{array}{ll}
\text { 1. } & \text { Orthonormality. } \\
\text { 2. } & \text { Filter length. } \\
\text { 3. } & \text { Vanishing order or moment. } \\
\text { 4. } & \text { Smoothness. } \\
\text { 5. } & \text { filter magnitude response } \\
\text { 6. } & \text { Group delay } \\
\text { 7. } & \text { Decomposition level. } \\
\text { 8. } & \text { Regularity. }
\end{array}
$$

A sufficient condition for the reconstruction of any signal $\mathrm{x}$ of finite energy by the formula

$$
\begin{aligned}
& W_{\varphi}\left(j_{0}, k\right)=\frac{1}{\sqrt{M}} \sum_{n} f(n) \varphi_{j_{0}, k}(n) \ldots \ldots \\
& W_{\psi}(j, k)=\frac{1}{\sqrt{M}} \sum_{n} f(n) \psi_{j, k}(n) \geq j_{0} \ldots
\end{aligned}
$$

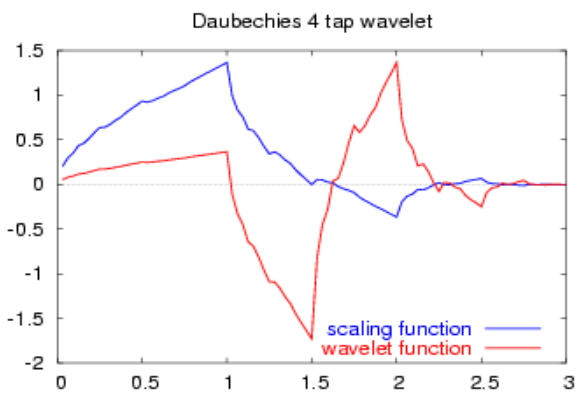

Fig.2: Daubechies4-functions

The complement inverse of the DWT is:

$$
f(n)=\frac{1}{\sqrt{M}} \sum_{k} W_{\varphi}\left(j_{0}, k\right) \varphi_{j_{0}, k}(n)+\frac{1}{\sqrt{M}} \sum_{j=j_{0}}^{\infty} \sum W_{\psi}(j, k) \psi_{j, k}(n)
$$

In this paper the fast wavelet transform is used both to analysis the image prior to segmentation enabling feature selection and made up smooth image. The Fast Wavelet Transform (FWT) is a computationally efficient implementation of the discrete wavelet transform (DWT) that exploits a surprising but fortunate relationship between the coefficients of the (DWF) and adjacent scales [3]. The (FWT) able to repeatedly decompose an image in the low frequency channels make it ideal for image analysis since the lower frequencies tend to dominate real images. By using wavelet transform it is easily to make up smooth image, the smooth image has strong component only in the low frequencies. In order to extract useful information from this it is useful to group the wavelet coefficients into channels representing the various frequency bands. Wavelets are effective instruments for smoothing images, using the wavelet transform with respect to second order symlets and zeroing the first level details coefficients of the original image's wavelet transform (and computing the modified transform's inverse). A two level wavelet decomposition of an image results seven main wavelet channels, as shown in figure 3 . The energy in these channels is quite differently. Images varies smoothly are heavily dominated by the low-frequency channel in their wavelet transforms, However the large energy is in the low frequency, these channels can be further grouped into lowfrequency (channels 1-4) and high-frequency (channels 5-7) bands. Hence the mean energy in the four low-frequency channels (1-4) is proposed as criterion for the segmentation of smooth images.

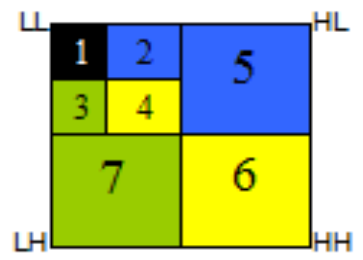

Fig.3: main channels of second level wavelet decomposition

Decomposition the image at the second level using fast wavelet transform will result seven main wavelet channels. To remove the high frequency that is available in the first level, we zeroing the first level details coefficients of the original image's wavelet transform, remaining four channels is contain the low-frequency channel in their wavelet transforms, and computing the modified transform's inverse, we will get the smooth image that is become easily to be segmented using means as a feature.

\section{CONVERT IMAGE SPACE}

In essence, a color model is a specification of a coordinate system and a subspace within that system where each color is represented by a single point. In this paper we convert the image from RGB space into NTSC space (also known as YIQ), color space enables us to quantify the visual differences in the image, in the NTSC format, image data consist of three components luminance $(\mathrm{Y})$, hue $(\mathrm{I})$, and saturation $(\mathrm{Q})$. One of the main advantages of this format is that the gray-scale information is separate from color data. All the color component in the "I" and "Q" components. The YIQ component are obtained from RGB component of the image using the transformation

$$
\left[\begin{array}{l}
Y \\
I \\
Q
\end{array}\right]=\left[\begin{array}{ccc}
0.299 & 0.587 & 0.114 \\
0.596 & -0.274 & -0.322 \\
0.211 & -0.523 & 0.312
\end{array}\right]\left[\begin{array}{l}
R \\
G \\
B
\end{array}\right]
$$

Note that element of the first row sum to one and the element of the next two rows sum to zero. This is as expected because for a gray-scale image all the RGB components are equal, so the I and Q components should be zero for such an image. 


\section{SEGMENTATION BY CLUSTERING}

One of the image segmentation literatures is Feature space clustering approaches [11]. The k-means clustering technique involve grouping together those pixies in the image whose feature vector represent point that are close together in the feature space. $\mathrm{K}$-means algorithm is an iterative technique that is used to partition an image into $\mathrm{K}$ clusters. The basic algorithm is:

$>$ Pick K cluster centers, either randomly or based on some heuristic.

$>$ Assign each pixel in the image to the cluster that minimizes the distance between the pixel and the cluster center.

$>$ Re-compute the cluster centers by averaging all of the pixels in the cluster.

$>$ Repeat steps 2 and 3 until convergence is attained (e.g. no pixels change clusters).

The final result is a number of clusters $\mathrm{k}$, where each hopefully depicts a perceptually different region in the image. Each cluster can be represented by the mean feature vector of all its members (pixels or block of pixels) known as its cluster center $C_{k}, k \in\{1, \ldots, k\}$. To obtain good segmentation, the number of each cluster should have feature vector lying as close as possible to the cluster centers, and the cluster center should be well separated.

Each pixel is labeled as being a member of the cluster center to which it is closest, using the distance functions $d\left(C_{k}, X\right)$, where $X$ is the pixel's feature vector. The distance function measures the distance between two points in the feature space and is simply the Euclidean distance metric here. Euclidean distance is the straight-line distance between two pixels. The cluster centers are then recalculated as the mean feature vector of all their members:

$$
C_{k}=\frac{1}{\left[g_{k}\right]} \sum_{S \in g_{k}} X_{S} \quad k \in\{1, \ldots, k\}
$$

Where

$$
g_{k} k \text { th cluster, }
$$

$S \quad$ Member of such a cluster,

$X_{S}$ Feature vector,

$\left[g_{K}\right]$ numbers of members in cluster $g_{k}$.

Pixels are then relabeled according to their new nearest cluster center, and the process is repeated until convergence to a sable partition of the feature space has occurred. This gives a segmentation result with a certain number of regions equal to the number of clusters $\mathrm{k}$. the clustering routine is repeated several time: once for every possible number of clusters. The difficulty then lies in determining the optimal number of clustering for the segmentation which is known as the true cluster number. Initially the distance is very high for only one cluster but decrease quickly as the number of clusters increases until the true cluster number is reached. They subsequently remain approximately constant or decrease very gradually. The true cluster number is thus taken to be the point at which the distance becomes approximately constant. However, this involves the use of a threshold that to set so that the optimal segmentation is always guaranteed.

\section{RESULTS}

The proposed algorithm was used to segment the RGB images, the image was segmented as shown in the figure:6 using different approaches, the first approach used wavelet features for the entire image by decomposition the entire image by using fast wavelet transform at level two resulted different subband channels that involve approximation coefficient and details coefficients (horizontal, vertical, and diagonal) in different levels, as smooth image that is easy to segment it by using the means as a feature as shown in figure 6(b), zeroing the first level details coefficients of the original image's wavelet transform, remaining four channels is contain the low-frequency channel in their wavelet transforms, and computing the modified transform's inverse. In the second approach we used the NTSC or color space as shown in figure 6(c) to quantify the visual differences in the image. In the third approach we used the $\mathrm{k}$-means clustering technique that is helped us to separate groups of objects, the objects are pixels with 'I' and 'Q' values, use k-means to cluster the objects into $\mathrm{K}$ clusters using the Euclidean distance metric. For every object in the input, k-means returns an index corresponding to a cluster. Label every pixel in the image with its cluster index as shown in figure 6(d). By using pixel labels, we separated objects in images by color, which resulted in four images in this example as shown in figure 6(e)-6(h).
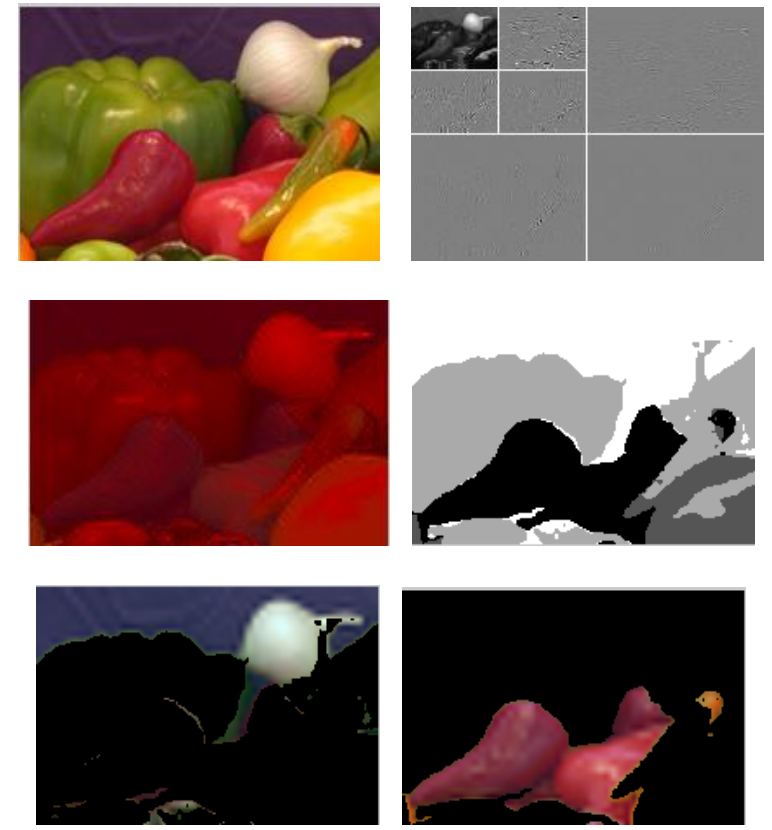

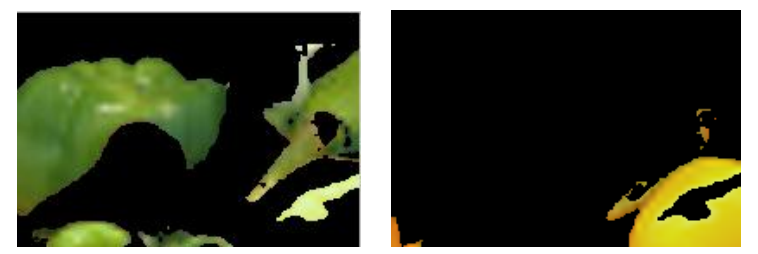

Fig.6: a) original image b) FWT at second level c) convert into NTSC space d) clustering e)-h) separated objects by color

\section{CONCLUSION}

Image segmentation routines too often concentrate only on some very application-specific segmentation of smooth regions. Many real or texture images are made up of smooth image, and the segmentation techniques must therefore incorporate features capable of describing these regions effectively. An algorithm that doses this by using wavelet analysis to select the relevant features for each area of the image prior to segmentation has been proposed here. A clustering routine is then invoked to perform the segmentation for the smooth areas of the images. One common difficulty with the k-mean clustering algorithm is the determination of the optimal number of region for segmentation: the so-called "true cluster number ". A completely automatic determination for true cluster number has been suggested here which, unlike existing methods, no threshold setting required.

\section{ACKNOWLEDGMENTS}

Praise be to God who enabled me to complete this project, without which it is not possible to be completed. I would like to offer sincere thanks to and my guide Dr. Ramesh R. Manza who helped me and gave me the confidence to complete this project. Special thanks to my parents and my wife and family who supported me a lot. Thank you very much for all.

\section{REFERENCES}

[1] L. P. Clarke et al., "MRI segmentation: Methods and applications," Magn. Resonance Imaging, Vol. 13, no. 3, pp. 343-368, 1995
[2] M. Kunt, A. Ikonomopoulos, and M. Kocher, "Secondgeneration image-coding techniques," proc. IEEE, Vol.73, no. 4, pp. 549-574, Apr. 1985.

[3] Rafael C. Gonzalez \& Richard E. Woods, AddisonWesley, "Digital Image Processing", 2002.

[4] A. K. Jain and F. Farrokhnia, "Unsupervised texture segmentation using Gabor filters," patt. Reogn., Vol. 24 no. 12, pp. 1167-1186, Dec. 1991.

[5] Y. Hu and T. J. Dennis, "texture image segmentations by context enhanced clustering," Proc. Inst. Elec. Eng.vision, Image, Signal processing, Vol. 114, no. 6, pp. 413-421, Dec. 1994

[6] I. Daubechies. Ten Lectures on Wavelets. Soc. Ind. Applied Math, Philadelphia, 1992.

[7] S. G. Mallat, "Multifrequency channel decomposition of images and wavelet models," IEEE Trans. Acoustics, speech, signal processing, Vol. 37, no. 12, pp. 20912110, Dec. 1989

[8] T. Chang and C.-C. J. Kao, "Texture analysis and classification with tree-structured wavelet transform," IEEE Trans. Image Processing, Vol. 2, no. 4, pp. 429441, Oct. 1993.

[9] subhasis Saha and Rao Vemuri “ Analysis based adaptive wavelet filter selection in Lossy image coding schemes", ISCAS-2000-IEEE international Symposium on Circuits and system May, Geneva, Switzerland, 2000.

[10] Bryan E. Usevitch " A Tutorial on Modern Lossy wavelet image compression: Foundations of JPEG 2000" ,IEEE signal processing Magazine, September,2001.

[11] D. Comaniciu and P. Meer. Mean shift: A robust approach toward feature space analysis. IEEE Trans. Pattern Anal. and Machine Intell., 24:603-619, 2002. 